\title{
Long-term non-invasive drug treatments for adult zebrafish
}

\author{
Yuting Lu ${ }^{1}$ and E. Elizabeth Patton ${ }^{1,2}$
}

1. MRC Human Genetics Unit and CRUK Edinburgh Centre, Institute of Genetics and Cancer, University of Edinburgh, Western General Hospital Campus, EH4 2XU, Edinburgh, UK.

2. Corresponding author: e.patton@ed.ac.uk 


\section{SUMMARY}

Zebrafish embryos are widely used for drug-discovery however administering drugs to adult zebrafish is limited by current protocols that can cause stress. Here, we develop a drug formulation and administration method for adult zebrafish by producing food-based drug pellets which are consumed voluntarily. We apply this to zebrafish with BRAF-mutant melanoma, a model that has significantly advanced our understanding of melanoma progression, but not of drug resistance due to the limitations of current treatment methods. Short-term, precise, and daily dosing with drug-pellets made with the BRAF ${ }^{\mathrm{V} 600 \mathrm{E}}$ inhibitor, vemurafenib, led to tumour regression. On-target drug efficacy was determined by phospho-ERK staining. Continued drug treatment led to the emergence, for the first time in zebrafish, of acquired drug resistance and melanoma relapse, modelling the responses seen in melanoma patients. This method presents a controlled, non-invasive approach that permits long-term drug studies, and can be widely applied to any adult zebrafish model. 


\section{SUMMARY STATEMENT}

This drug-pellet approach is a precise dosing method to administer short or long-term drug treatments to adult zebrafish, that is stress-free for the fish and requires minimal animal handling. We use this method to develop new drug-resistant models of melanoma in zebrafish, opening new doors for modelling and screening drug treatments in adult zebrafish.

\section{KEYWORDS}

adult zebrafish, drug pellets, non-invasive, long-term treatment, melanoma, vemurafenib, melanoma regression, drug resistance models 


\section{INTRODUCTION}

Over the past two decades zebrafish have emerged as an important model for drug discovery, directly leading to drugs that have entered clinical trials or for compassionate use (Patton et al., 2021b). These include therapies that promote hematopoietic stem cell renewal (North et al., 2007), prevent antibiotic-induced ototoxicity (Kitcher et al., 2019), and those that treat childhood epilepsy (Baraban, 2021), cancer (Mandelbaum et al., 2018; White et al., 2011; Yan et al., 2019), lymphatic anomaly ( $\mathrm{Li}$ et al., 2019), arteriovenous malformation (Al-Olabi et al., 2018), and fibrodysplasia ossificans progressive (Yu et al., 2008), among others. This success is, in part, because zebrafish are vertebrates and share over $80 \%$ of disease genes with humans (Howe et al., 2013), as well as shared drug metabolism, physiology and pharmacology (MacRae and Peterson, 2015). Thus, zebrafish pre-clinical disease models are important platforms for drug discovery and repurposing, even at times leading to new treatment strategies for patients directly from zebrafish models.

With some exceptions, most zebrafish drug discovery, gene-drug screens and compoundphenotype evaluation studies are performed using zebrafish embryos. However, embryos and larval stages do not fully recapitulate adult disease states and lack a complete immune system. Drug screening and discovery in adult zebrafish - and modelling the impact of long-term drug treatment - has been limited by methods of drug administration, which can be complex, harmful, and imprecise. Current methods for experimental drug discovery in adult zebrafish involve adding the drug to the fish water which can irritate exposed mucosal surfaces (e.g. eyes, gills), is not appropriate for water-insoluble compound administration, and involves administrating large quantities of drug to fish water with unknown final drug concentrations absorbed by the fish. Alternatively, drugs can be administered by oral gavage or through injection (retro-orbital or intraperitoneal), which are more precise for dosing, but invasive and sometimes fatal, and require repeated anesthesia (Dang et al., 2016; Kinkel et al., 2010; Pugach et al., 2009). While these 
methods are generally acceptable for short-term drug treatments, they are problematic for longer term drug protocols because they can lead to accumulative distress and injury to the animal. Adding antibiotics to jelly like food has been used to manage zebrafish colony health (Chang et al., 2017), however, these methods were not designed to administer precise drug treatments to individual fish in the experimental and pre-clinical disease model context, and are therefore not appropriate to investigate dose-based drug responses. Thus, non-invasive and precise drug delivery protocols that permit longitudinal experimental drug treatments for adult zebrafish are not well developed.

Our laboratory uses zebrafish to model the progression of melanoma, the deadliest form of skin cancer (Travnickova and Patton, 2021). Zebrafish melanoma models have provided key insights into the origins, progression and new drug targets for melanoma (Baggiolini et al., 2021; Ceol et al., 2011; Kaufman et al., 2016; Patton et al., 2005; Travnickova et al., 2019; Venkatesan et al., 2018; White et al., 2011). However, a significant gap in the field has been to generate zebrafish melanoma models that recapitulate the development of acquired drug resistance as seen in patients (Patton et al., 2021a). Indeed, acquired resistance is one of the major challenges limiting the progression-free survival time of melanoma patients on therapies that specifically target BRAF $^{\mathrm{V} 600 \mathrm{E}}$ and MEK signalling (Chapman et al., 2011; Larkin et al., 2014; Long et al., 2015; Luke et al., 2017; Ribas et al., 2014; Robert et al., 2015; Sosman et al., 2012). This gap is unfilled in zebrafish melanoma models due to the lack of sustainable long-term drug administration methods for adult fish, despite evidence for the strong potential of zebrafish models recapitulating many human melanoma plasticity states, including residual disease (Travnickova and Patton, 2021).

Here, we present a novel drug formulation and administration method for adult zebrafish which enables the delivery of precise drug concentrations directly via food pellets. As a proof-ofprinciple, we fed zebrafish with $\mathrm{BRAF}^{\mathrm{V} 600 \mathrm{E}}$ melanomas pellets containing vemurafenib and showed that they caused immediate and on-target efficacy in reducing melanoma growth. Long- 
term treatments (>2 months / daily treatment) led to drug resistance and melanoma progression, enabling zebrafish models of melanoma drug resistance for the first time. Our drug pellet method opens new doors to model the effects of drug dose, administration, and long-term treatments in adult zebrafish that is non-invasive and limits animal handling, and is widely applicable across a wide range of zebrafish disease models.

\section{RESULTS}

\section{Formulation of drug-pellets for adult zebrafish}

We wanted to generate drug pellets that would deliver consistent drug doses to treat melanoma while also being quickly and freely consumed by the zebrafish. To begin, we prepared vemurafenib pellets using a drug-pellet mould that we designed and 3D-printed, so that each drug pellet would be $2 \mathrm{~mm}$ in diameter (comparable to the size of a zebrafish egg, and which are easily consumed by adults) (Figure 1A). We suspended dry fish food in water, and mixed this with agaragar and gelatine powder to create a food paste to generate the base of the food pellet. To prepare the pellets, $10 \mathrm{~cm}^{3}$ dry fish food was added to water up to $50 \mathrm{ml}$ in a conical centrifuge tube, completed with $1 \mathrm{~g}$ of food-grade agar-agar plus $2 \mathrm{~g}$ of gelatine powder, and shaken well to generate a red-coloured mix (Figure 1B). This recipe was optimised to achieve a balance between congelation for laboratory handling and a palatable texture for the fish. The mixture was then transferred into a $100 \mathrm{ml}$ borosilicate container and microwaved for $\sim 1 \mathrm{~min}$ to reach boiling, turning the mixture into a smoothie-like texture (Figure 1B). Just before the mixture congealed, we added the desired drug (i.e. vermurafenib) or DMSO as a control (Figure 1A). We prepared our vemurafenib pellets to each deliver $100 \mathrm{mg} / \mathrm{kg}$ vemurafenib (as shown to induce tumour regression when administered by oral gavage)(Dang et al., 2016). Briefly, $10 \mathrm{mg}$ of vemurafenib 
powder was resuspended in $300 \mu \mathrm{LMSO}$ and then mixed well with $700 \mu \mathrm{l}$ agar-fish food mixture while warm in a $1.5 \mathrm{ml}$ microcentrifuge tube, generating a pink-coloured paste (Figure 1C). Each well of the 3D-printed pressing mould is $5 \mu \mathrm{l}$, and therefore each pellet contained $0.05 \mathrm{mg}$ vemurafenib. Given that the average weight of each fish is $0.5 \mathrm{~g}$, one vemurafenib pellet per day will deliver the dose of $100 \mathrm{mg} / \mathrm{kg}$.

A sheet of parafilm large enough to cover the 3D-printed drug pressing mould and an appropriately sized plastic roller were prepared as tools to press drug pellets (Figure 1D). Once the food-drug paste began to congeal, the paste was transferred onto the drug-pressing mould, covered with the parafilm sheet (to form a "sandwich" with the mould between the parafilm sheet and the backing paper), and then pressed into the mould with the plastic roller (Figure 1E). The parafilm sheet was lifted, and the mould carefully removed to reveal the drug pellets now formed and adhering to the backing paper (surface tension will trap most of the drug pellets neatly on the backing paper) (Figure 1E-G). This approach enables batches of >100 cylindrical tablet-like drug pellets to be conveniently made during a single preparation. DMSO control pellets were prepared in a similar way. In this experiment, drug pellets were prepared once every week and stored at $80^{\circ} \mathrm{C}$ as individual pellets in PCR tubes for daily dose aliquots (Figure 1H). The entire process of pressing drug pellets is shown in Video 1.

\section{Administration of drug-pellets to adult zebrafish by free feeding}

We then fed the drug-pellets to individual adult zebrafish with melanoma that were singly-housed in $1 \mathrm{~L}$ tanks. As shown in Video 2, zebrafish actively sought for and consumed the drug pellet voluntarily. In general, zebrafish consumed their drug pellets immediately, however, any pellets that were ignored for more than 15 minutes were removed and replaced by fresh pellets to ensure 
consistent dosing in drug administration. Using a pipette to gently deliver the drug pellets encouraged zebrafish to spot and consume the pellets. We observed no distress or toxicity in the fish from either the drug-pellet formulation or the administration.

\section{Vemurafenib drug-pellets reduce zebrafish BRAF ${ }^{\mathrm{V} 600 \mathrm{E}}$ melanoma burden}

To test the drug efficacy, we provided DMSO or vemurafenib drug pellets to adult zebrafish with BRAF $^{\mathrm{V} 600 \mathrm{E}}$ p53 mutant melanomas (Patton et al., 2005) and compared the tumour responses between the two groups. To track the tumour size over time, we imaged and analysed the brightfield images of each tumour from these individuals every week (Figure 2A). DMSO treated zebrafish showed continuous lesion expansion and tumour growth (Figure 2B, 2C), while 100 $\mathrm{mg} / \mathrm{kg}$ vemurafenib treated fish showed tumour regression over 3 weeks (Figure 2D, 2E), with the average tumour size reduced by $60 \%$ in 2 weeks, and $70 \%$ in 3 weeks, compared to the pretreatment state (Figure 2E). This result is highly comparable to the observation from the previous $100 \mathrm{mg} / \mathrm{kg}$ oral-gavage regime (Dang et al., 2016), from which they reported 2-week daily treatment reduced the melanoma size by $70 \%$ in average.

Next, we increased the dose of vemurafenib by feeding the fish 2 drug-pellets per day, and observed a higher proportion of lesions had improved regression ( 4 out 9 reduced by $>80 \%$ in 3 weeks, compared to 1 out 6 in fish treated with 1 pellet/day). Thus, drug-pellets are highly effective at treating zebrafish melanoma, well tolerated, and can be used in dose escalation studies. 


\section{Long-term drug treatments lead to drug resistance}

Patients with BRAF mutant melanoma and receiving targeted therapy will often show a dramatic reduction in melanoma burden, followed by recurrent melanoma growth from residual disease (or persister cells)(Marine et al., 2020; Shen et al., 2020b). We and others have shown that persister cell states are heterogeneous and consist of cell states that pre-exist in the primary tumour and emerge de novo (Rambow et al., 2018; Travnickova et al., 2019). We have studied these states in zebrafish by conditional expression of the master melanocyte transcription factor MITF, while others have used mouse xenograft studies to follow human melanoma cells following BRAF inhibitors (Rambow et al., 2018; Shen et al., 2020a; Travnickova et al., 2019). However, there are no zebrafish models of BRAF inhibitor resistance over time because of the limitations of long-term drug delivery methods to adults.

We noticed that one tumour developed resistance and regrowth at the end of week 3 on treatment at $200 \mathrm{mg} / \mathrm{kg}$ in the short-term treatment protocol (Figure 2G; Tumour 4). We reasoned that drugpellets could be used to investigate the effect of long-term vemurafenib treatment on BRAF ${ }^{\mathrm{V} 600 \mathrm{E}}$ zebrafish melanoma, and generate models of drug-resistance. Similar patterns of tumour responses were observed in mouse xenograft models which showed that human melanoma tumours regressed following daily MAPK-inhibitor treatment, and then entered a stable or residual disease stage around 18 days, followed by recurrent growth as drug-resistant tumours around 50 days (Rambow et al., 2018; Wang et al., 2018).

We first treated zebrafish on the course of $100 \mathrm{mg} / \mathrm{kg}$ vemurafenib daily pellets, and followed the tumour response over 5 weeks (Figure 3A, B). In our experiment, we noticed that by 4 weeks at $100 \mathrm{mg} / \mathrm{kg}$, the melanomas began to regrow, so we increased the dose to $200 \mathrm{mg} / \mathrm{kg}$ at week 5 (Figure 3B). We found that melanomas were initially responsive to the increased vemurafenib concentration, but that they again began to regrow by 8 weeks (Figure 3B). Next, we treated a 
cohort of zebrafish with melanomas with $200 \mathrm{mg} / \mathrm{kg}$ vemurafenib for four weeks, and found that the melanomas responded rapidly to the treatment, with drug resistance and progressive disease observed as early as 4 weeks (Figure 3C, 3D). These studies indicate that our drug-pellet delivery methods can model long-term treatment response adaptation, from the initial melanoma regression, through to stable disease, and finally recurrent and drug resistant disease.

\section{Validation of drug-pellet efficacy in adult zebrafish cancer}

To assess the on-target efficacy of vemurafenib to inhibit BRAF ${ }^{\mathrm{V} 600 \mathrm{E}}$ activity in zebrafish melanoma, we performed immunofluorescence staining on melanoma sections to assess the MAPK pathway activity using phospho-Erk1/2, a downstream target of activated BRAF signalling. Melanoma samples collected from the early-responding stage of vemurafenib pellet treatment (weeks 2 and 3 ) had significantly reduced levels of phospho-Erk1/2 compared to DMSO treated control samples (Figure 4A, 4B), indicating that vemurafenib pellets have sufficient bio-availability to target $\mathrm{BRAF}^{\mathrm{V} 600 \mathrm{E}}$ in melanoma and to lead to melanoma regression.

Next, we analysed tumours that showed melanoma recurrence on vemurafenib pellets, and found that although the responses were varied, on average, tumours increased levels of phosphoErk1/2, consistent with that seen in patients (Manzano et al., 2016; Proietti et al., 2020) (Figure 4A, 4B). Total Erk1/2 levels measured by immunofluorescence staining showed no significant changes across all samples (Figure 4C). These results validate the on-target efficacy of the drug compound delivered by our pellet feeding method. 


\section{DISCUSSION}

Zebrafish are a powerful model system for drug discovery, but while drug treatments for embryos and larvae can be easily administered through the water, drug discovery in adult zebrafish is limited by a lack of efficient, non-invasive and long-term permissive drug administration methods. Here, we provide a new method to generate drug pellets that can be easily fed to adult zebrafish to administer a controlled and precise drug dose in a non-invasive process. We apply this method to our zebrafish BRAF mutant melanoma models (Patton et al., 2005) and demonstrate that the melanomas respond to vemurafenib drug-pellet therapy. We validate the on-target efficacy of the drug by showing a reduction in total phospho-Erk1/2 in the melanoma following treatment. Longterm studies (>2 months) demonstrate that upon drug treatment, zebrafish melanomas undergo regression followed by recurrent disease, as seen in patients (Marine et al., 2020; Shen et al., 2020b; Travnickova and Patton, 2021). For the first time, this method enables us to model longterm melanoma drug treatment and resistance stages in zebrafish genetic melanoma models, an immunocompetent model system.

We found no toxicity or side-effects from the drug-pellet method, indicating that this method supports the tenets of the 3Rs (Replacement, Reduction and Refinement) in Animal Research (https://nc3rs.org.uk/). Specifically, by reducing the animal handling and total exposure required for drug administration, our method is a Refinement for drug delivery because it minimises zebrafish stress and improves welfare.

In conclusion, we provide a drug-pellet method to administer precise doses of drugs to adult zebrafish in a non-invasive, free-feeding based procedure. The drug-pellets can be individually frozen so that experiments can be controlled for batch effects, are suitable for drugs with low solubility in water (such as vemurafenib, which is hydrophobic), and provides a platform for drug combinations and screens. For a broader spectrum of application, pellet size and number can be 
adapted easily through modifying the drug-press 3D printing mould and modifying the quantities of the food paste recipe. While our experiments here focus on cancer studies in zebrafish, we expect this method will be applicable to a wide range of zebrafish disease models, and opens new doors for drug discovery in the complex biological context of adult zebrafish. 
RESOURCES TABLE

\begin{tabular}{|c|c|c|}
\hline REAGENT or RESOURCE & SOURCE & IDENTIFIER \\
\hline \multicolumn{3}{|l|}{ Antibodies and Chemicals } \\
\hline $\begin{array}{l}\text { Phospho-p44/42 MAPK (Erk1/2) (Thr202/Tyr204) } \\
\text { Antibody }\end{array}$ & Cell Signaling & Cat No: \#9101 \\
\hline p44/42 MAPK (Erk1/2) Antibody & Cell Signaling & Cat No: \#9102 \\
\hline $\begin{array}{l}\text { Goat anti-Rabbit lgG }(\mathrm{H}+\mathrm{L}) \text { Highly Cross-Adsorbed } \\
\text { Secondary Antibody Alexa Fluor } \AA 488 \text { conjugate }\end{array}$ & $\begin{array}{l}\text { Thermo Fisher } \\
\text { Scientific (Life } \\
\text { Technologies) }\end{array}$ & Cat No: A-11034 \\
\hline Vemurafenib (PLX4032) & SelleckChem & Cat No: S1267 \\
\hline Dimethyl sulfoxide (DMSO) & SigmaAldrich & Cat No: $67-68-5$ \\
\hline \multicolumn{3}{|l|}{ Commercial Food Ingredient } \\
\hline ZM Flakes & $\begin{array}{l}\text { ZM Fish Food } \\
\text { and Equipment }\end{array}$ & $\mathrm{N} / \mathrm{A}$ \\
\hline ZM Medium Premium Granular & $\begin{array}{l}\text { ZM Fish Food } \\
\text { and Equipment }\end{array}$ & $\mathrm{N} / \mathrm{A}$ \\
\hline ZM Small Granular & $\begin{array}{l}\text { ZM Fish Food } \\
\text { and Equipment }\end{array}$ & $\mathrm{N} / \mathrm{A}$ \\
\hline Hikari Micro Pellets & $\begin{array}{l}\text { Kyorin Food } \\
\text { Industries, Ltd. }\end{array}$ & $\mathrm{N} / \mathrm{A}$ \\
\hline Cooks' Ingredients Agar Agar & $\begin{array}{l}\text { Waitrose \& } \\
\text { Partners }\end{array}$ & $\mathrm{N} / \mathrm{A}$ \\
\hline Dr.Oetker Gelatine & Dr. Oetker KG & $\mathrm{N} / \mathrm{A}$ \\
\hline \multicolumn{3}{|l|}{ Experimental Models: Organisms/Strains } \\
\hline $\operatorname{Tg}\left(\right.$ mitfa:BRAF $\left.{ }^{V 600 E}\right), p 53^{M 214 K}$ & $\begin{array}{l}\text { Patton et al. } \\
(2005)\end{array}$ & $\begin{array}{l}\text { ZFIN Cat \#: ZDB- } \\
\text { TGCONSTRCT- } \\
\text { 070117-106, ZDB- } \\
\text { ALT-050428-2 }\end{array}$ \\
\hline \multicolumn{3}{|l|}{ Software and Algorithms } \\
\hline TinkerCAD & $\begin{array}{l}\text { https://www.tinker } \\
\text { cad.com/ }\end{array}$ & $\mathrm{N} / \mathrm{A}$ \\
\hline Ultimake Cura & $\begin{array}{l}\text { https://ultimaker.c } \\
\text { om/software/ultim } \\
\text { aker-cura }\end{array}$ & RRID:SCR_018898 \\
\hline GraphPad Prism & $\begin{array}{l}\text { http://www.graph } \\
\text { pad.com/ }\end{array}$ & RRID:SCR_002798 \\
\hline Fiji 1.0 & http://fiji.sc & RRID:SCR_002285 \\
\hline
\end{tabular}




\section{METHODS}

\section{Zebrafish maintenance and husbandry}

Zebrafish were maintained in accordance with UK Home Office regulations, UK Animals (Scientific Procedures) Act 1986, under project license P8F7F7E52. All experiments were approved by the Home Office and AWERB (University of Edinburgh Ethics Committee).

\section{Zebrafish melanoma models}

Zebrafish were genotyped using DNA extracted from fin clipped tissue by PCR to establish the mutant allele status tp53 ${ }^{M 214 K}$ or mitfa-BRAF ${ }^{V 600 E}$ as described in our previous publications (Travnickova et al., 2019). The emergence of melanoma is usually observed in individuals aged 3- to 6-month-old. Individuals used for DMSO versus vemurafenib drug pellets treatment in this experiment were siblings and were aged 5- to 6-month-old when entering the treatment scheme.

\section{Drug pellets ingredients}

The recipe of our routine dry fish food mix consists of ZM flakes, ZM Medium Premium Granular, ZM Small Granular and Hikari MicroPellets mixed at a ratio of 2:3:2:5. Food-grade agar-agar or gelatine powder were purchased from local grocery stores. Vemurafenib (SelleckChem, CAS\#918504-65-1) powder were resuspended in DMSO before mixing with fish-food paste as described.

\section{D-printing}

The 3D-modelling and design of the drug-pressing mould was carried out on Tinkercad

(Supplementary File 1) followed by slicing set-up using Ultimaker Cura and 3D-printed via Ultimaker 3 with AA 0.25 generic PLA. 


\section{Imaging of adult zebrafish and tumour size measurement}

Fish were briefly anesthetised (Tricaine in PBS 1:10,000 concentration) once every week for imaging purposes to follow the tumour burden changes during the experiment. Each fish was anaesthetised for no longer than 10 min per session and fully recovered in fresh system water. Brightfield images were taken for each fish positioned on both sides. Images of fish lesions were captured at the same magnification scale at the same microscope every week. The size of each lesion was quantified by using the manual field selection in Fiji on each tumour image, then compared to the matching pre-treatment lesion to calculate the relative percentage change. Lesions that could be observed from both sides of the fish were measured by combining the area number averaged from both sides.

\section{Zebrafish histology and IHC quantification}

Zebrafish melanoma samples were collected, fixed, and processed as described in our earlier publications (Lister et al., 2014). MAPK activity was assessed using phospho-p44/42 MAPK (ERK1/2) (Thr202/Tyr204) primary antibody (1:200, rabbit, Cell Signaling Technologies \#9101), total p44/42 MAPK (ERK1/2) primary antibody (1:200, rabbit, Cell Signaling Technologies \#9102), and Alexa-fluor 488 secondary antibody (1:1000, goat-anti-rabbit IgG, Life Technologies \#A11034). Nuclei were stained with DAPI dye (1:1000, Life Technologies \#62248). 


\section{ACKNOWLEDGEMENTS}

We are grateful to Cameron Wyatt and the IGC Zebrafish Facility for zebrafish management and husbandry, the IGC Imaging Facility for supporting the imaging experiments, and Helen Caldwell and Elaine McLay for histology. We appreciate the constructive inputs from Cameron Wyatt and Jana Travnickova during the development of this project. We are grateful for uCreate Studio team of the University of Edinburgh for providing the equipment and materials for the 3D-printing selfservice. EEP is funded by MRC HGU Program (MC_UU_00007/9), the European Research Council (ZF-MEL-CHEMBIO-648489), and Melanoma Research Alliance (687306).

\section{AUTHOR CONTRIBUTIONS:}

Conceptualization: YL; Methodology: YL; Validation: YL; Formal analysis: YL; Investigation: YL;

Resources: YL, EEP; Writing original draft: YL, EEP; Writing review and editing: YL, EEP; Visualization: YL, EEP; Supervision: EEP; Funding acquisition: EEP.

\section{DECLARATION OF INTERESTS}

E.E.P. is the Editor-in-Chief at Disease Models \& Mechanisms but is not included in any aspect of the editorial handling of this article. 


\section{FIGURE TITLES}

Figure 1. Technical setup and drug pellet preparation.

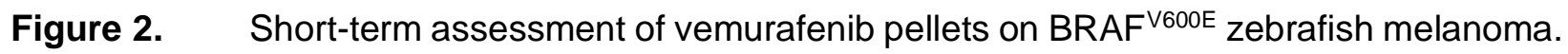

Figure 3. Long-term vemurafenib drug pellet treatment causes acquired drug resistance in zebrafish melanoma.

Figure 4. On-target efficacy of vemurafenib drug pellet treatment

\section{VIDEO LEGENDS}

Video 1. Preparation of drug pellets.

Drug supplemented food-agar mixture paste was cooled in a petri-dish, and once partially congealed, the jelly-like paste was transferred onto the 3D-printed mould and pressed into pellets between the parafilm sheet and backing paper.

\section{Video 2. Free-feeding adult zebrafish consume drug pellets.}

Single-housed adult zebrafish were fed once daily with artemia during the day and given drugpellets in the late afternoon. The video shows how zebrafish actively sought for and consumed the drug pellet voluntarily without any handling. 
A

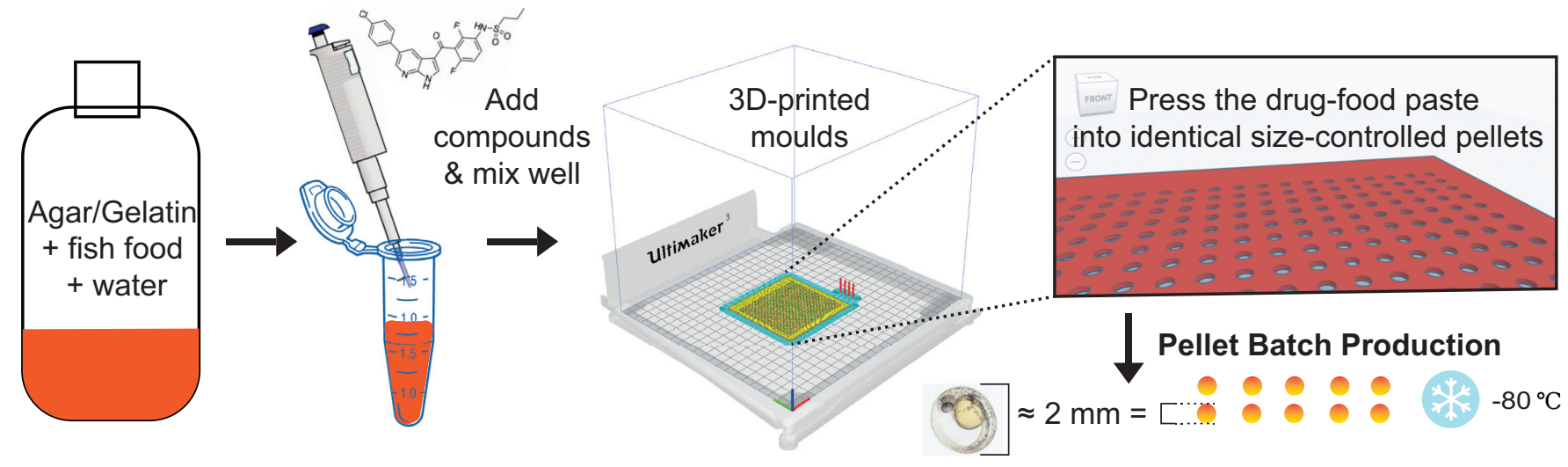

B

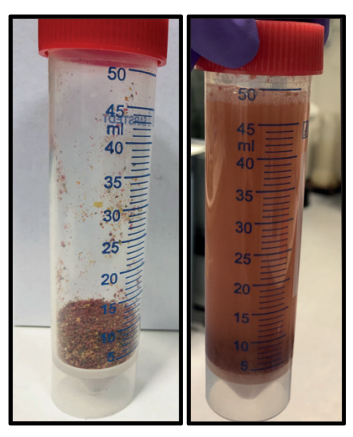

C

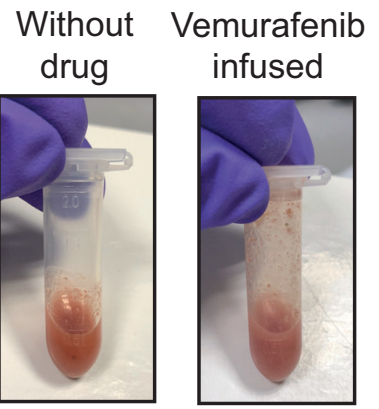

D

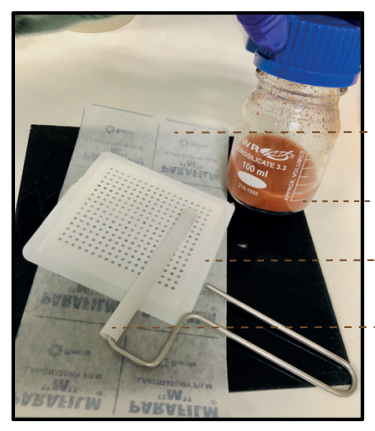

A parafilm Sheet

Heated gelatin \& fish food broth 3D-printed drug pressing mould A plastic roller

E
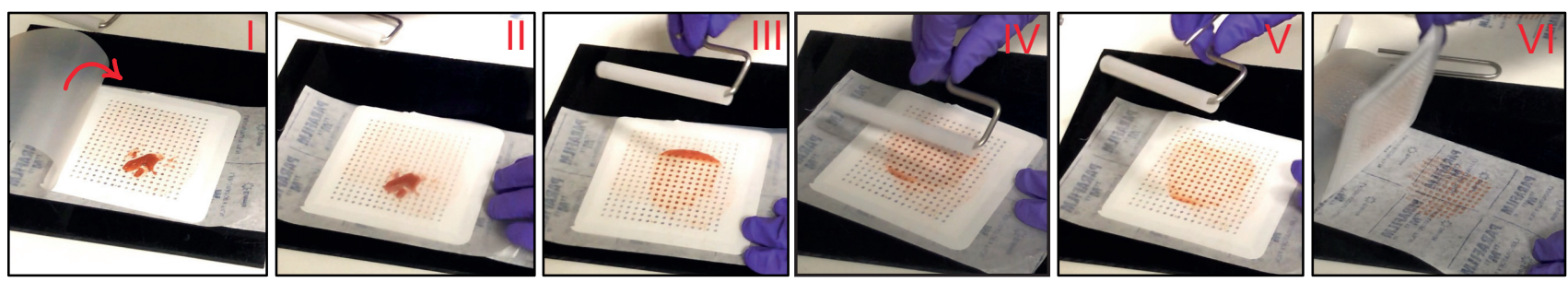

$\mathbf{F}$

G

H
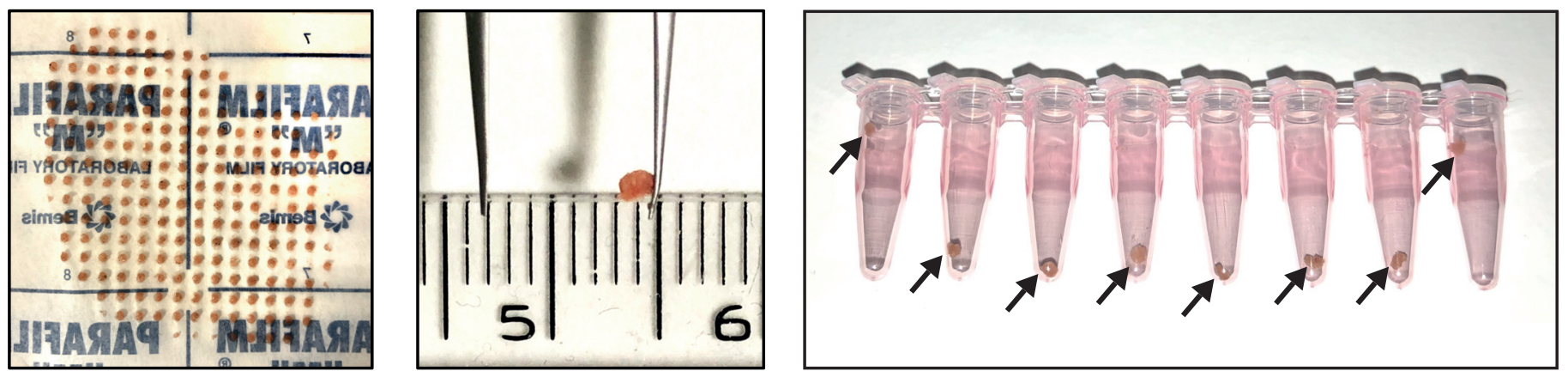
Figure 1. Technical setup and drug pellet preparation.

A. Schematic overview of the drug-pellets formulation and manufacturing pipeline.

B. Dry fish food mix and the food-agar mixture resuspended in water.

C. The red-colour food-agar paste becomes pink once supplemented with vemurafenib dissolved in DMSO.

D. The tools used for pressing drug-pellets in the mould.

E. Sequential series of photos (I-VI) showing the process of drug-pellet pressing. The parafilm sheet is peeled from the backing paper, and the mould is placed on the backing paper. The drug-paste is applied on to the mould (I), and the parafilm sheet is gently lowered to cover the paste and mould (II). Next, using the roller, the drug-paste is evenly applied into the holes of the mould (III-V). The parafilm sheet is lifted, followed by carefully removing the mould, and the drug pellets adhere to the backing paper ( $\mathrm{VI})$.

F. A freshly prepared batch of drug-pellets. Surface tension retains the pellets on the parafilm backing paper.

G. A drug-pellet recovered from $-80^{\circ} \mathrm{C}$ storage, maintaining the flat-cylinder shape. The ruler shown in the picture is scaled in $\mathrm{cm} / \mathrm{mm}$.

H. Drug-pellets aliquoted into daily doses per fish in PCR tubes, ready for $-80^{\circ} \mathrm{C}$ storage, with arrows highlighting the drug-pellets inside the tubes. 
bioRxiv preprint doi: https://doi.org/10.1101/2021.12.21.473492; this version posted December 23, 2021. The copyright holder for this preprint (which was not certified by peer review) is the author/funder. All rights reserved. No reuse allowed without permission.

A

B

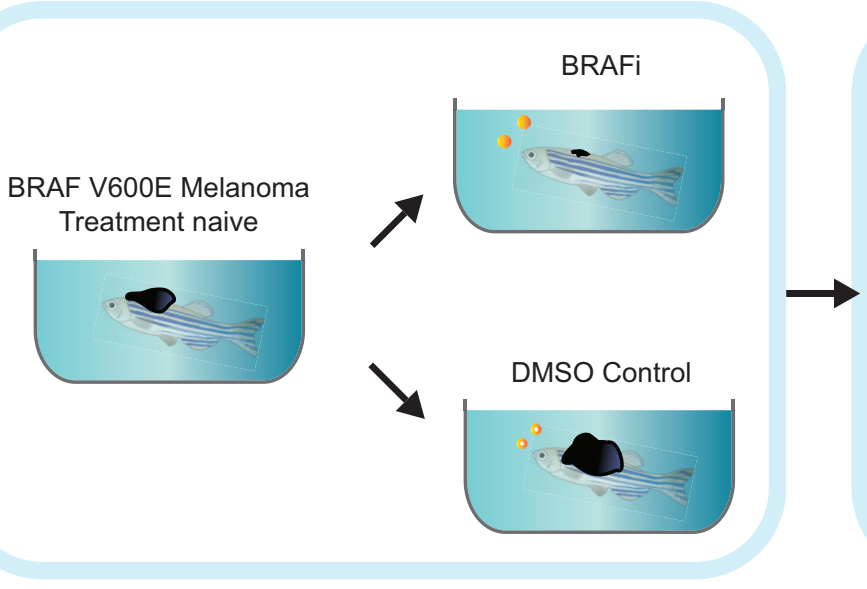

Pre-treatment
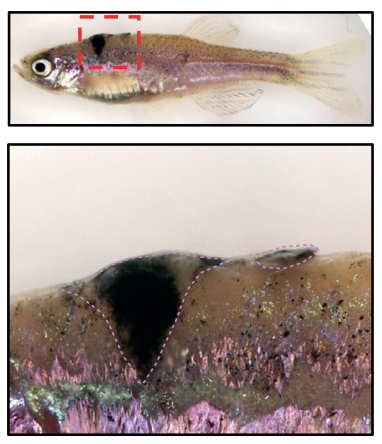

D

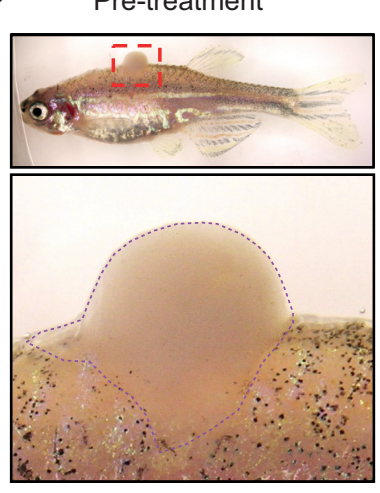

$\mathbf{F}$

Pre-treatment
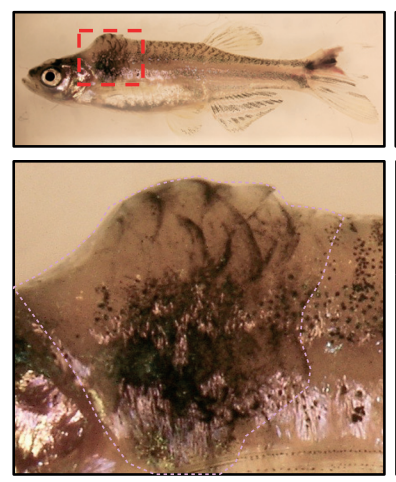

DMSO Week 1
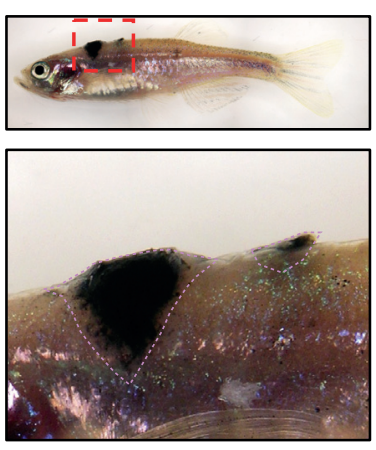

$100 \mathrm{mg} / \mathrm{kg}$ BRAFi Week 1

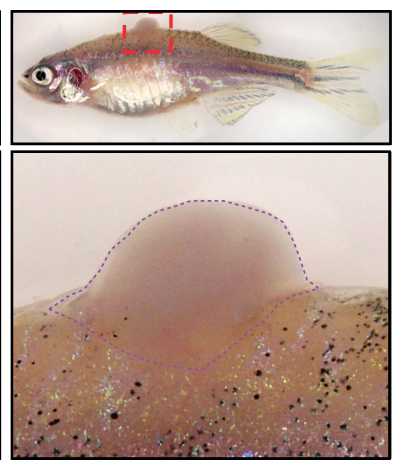

$200 \mathrm{mg} / \mathrm{kg}$ BRAFi Week 1
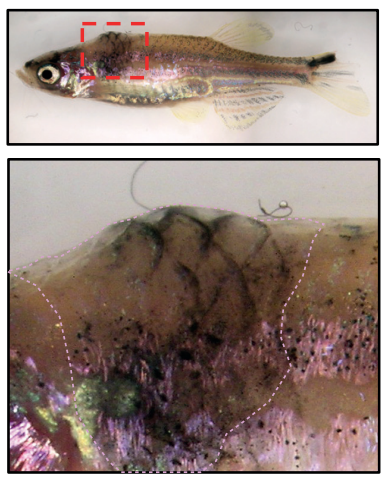

$100 \mathrm{mg} / \mathrm{kg}$ BRAFi Week 3
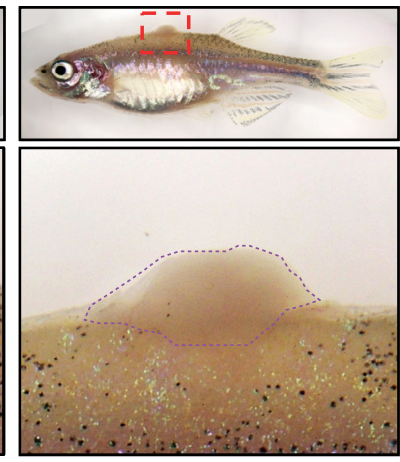

$200 \mathrm{mg} / \mathrm{kg}$ BRAFi Week 3
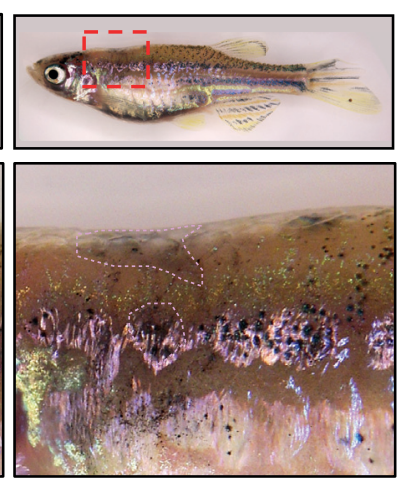

Temporary Anaesthesia Brightfield Imaging
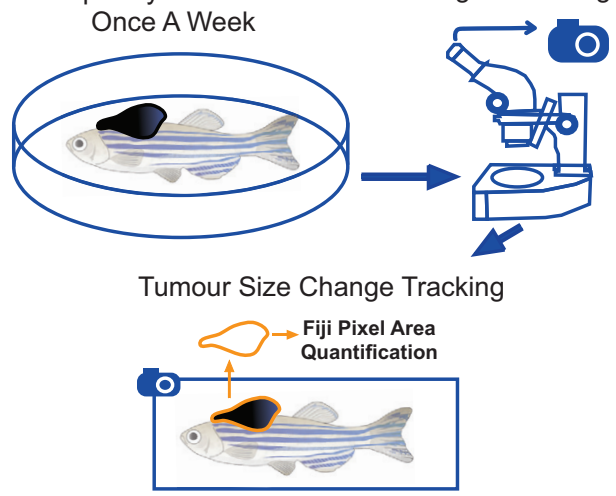

C

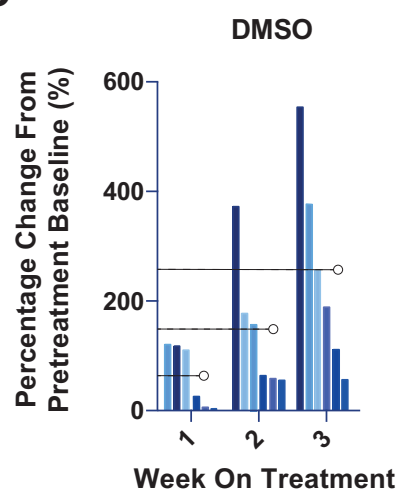

E

$100 \mathrm{mg} / \mathrm{kg}$ Vemurafenib

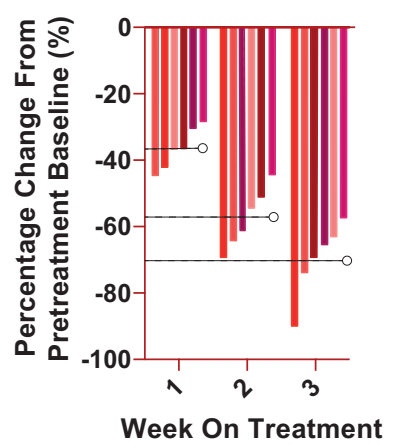

$\mathbf{G}$

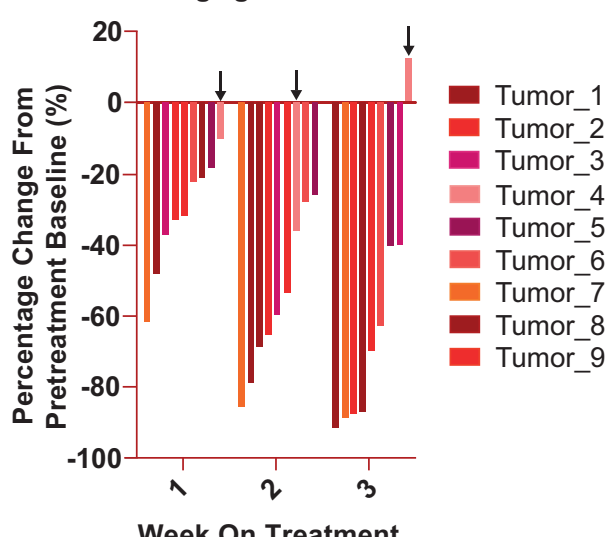

Tumor_1

- Tumor_2

- Tumor_3

Tumor_4

- Tumor_5

Tumor_6

Average
Week On Treatment 


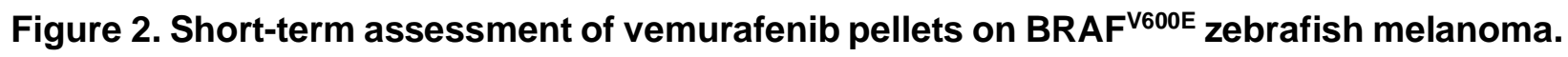

A. Schematic overview of drug-pellet free feeding administration, tumour response tracking, and evaluation for each fish treated with vemurafenib or DMSO pellets.

B. Representative images of $\mathrm{BRAF}^{\mathrm{V} 600 \mathrm{E}}$ zebrafish melanoma progressing on treatment of DMSO pellets. Zoomed regions are indicated by red dashed boxes. Dotted line outlines the melanoma.

C. Quantification of melanoma size change each week (by percentage) on DMSO pellets, comparing to the lesion imaged on the day pre-treatment. Fish receiving DMSO pellets $(\mathrm{N}=4$; tumour counts $n=6$ ). Average of tumour size changes indicated by dashed lines.

D. Representative images of $\mathrm{BRAF}^{\mathrm{V} 600 \mathrm{E}}$ zebrafish melanoma regressing while treated with 100 $\mathrm{mg} / \mathrm{kg}$ vemurafenib pellets. Dotted line outlines the melanoma.

E. Quantification of melanoma size change each week on $100 \mathrm{mg} / \mathrm{kg}$ pellets by percentage, compared to pre-treatment. Fish receiving $100 \mathrm{mg} / \mathrm{kg}$ vemurafenib pellets $\mathrm{N}=3$. Lesion counts $n=6$. Average of lesion size changes indicated by dashed lines.

F. Representative images of $\mathrm{BRAF}^{\mathrm{V} 600 \mathrm{E}}$ zebrafish melanoma regressing on treatment of 200 $\mathrm{mg} / \mathrm{kg}$ vemurafenib pellets.

G. Quantification of melanoma size change each week on $200 \mathrm{mg} / \mathrm{kg}$ pellets by percentage, comparing to the lesion imaged on the day pre-treatment. Fish receiving $200 \mathrm{mg} / \mathrm{kg}$ vemurafenib pellets $N=4$. Lesion counts $n=9$. One lesion (Tumour 4) with regrowth observed by week 3 on $200 \mathrm{mg} / \mathrm{kg}$ vemurafenib pellets is indicated by arrows. 
bioRxiv preprint doi: https://doi.org/10.1101/2021.12.21.473492; this version posted December 23, 2021. The copyright holder for this preprint (which was not certified by peer review) is the author/funder. All rights reserved. No reuse allowed without permission.

A

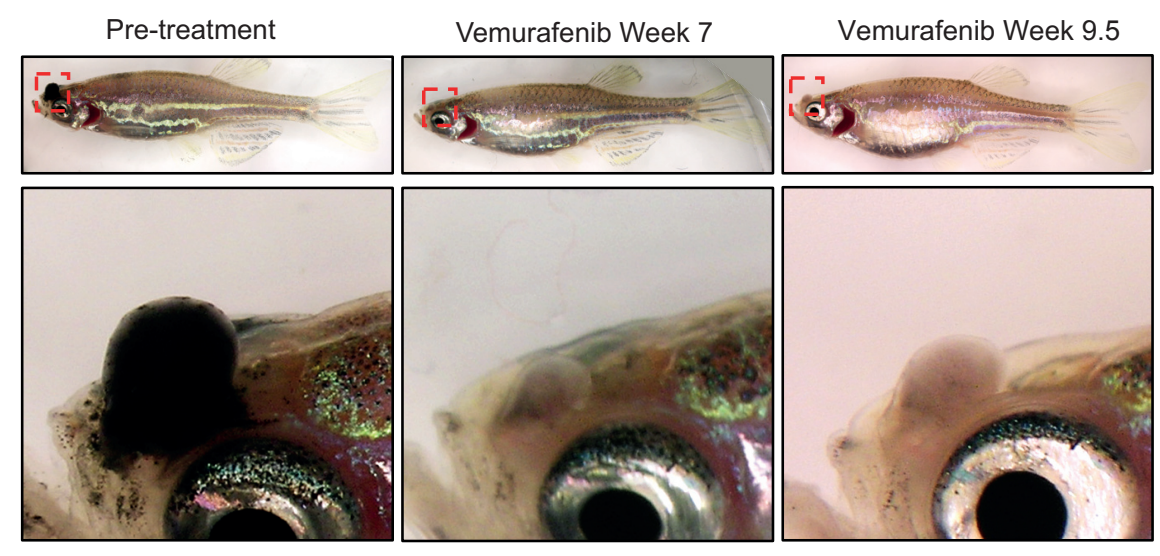

C
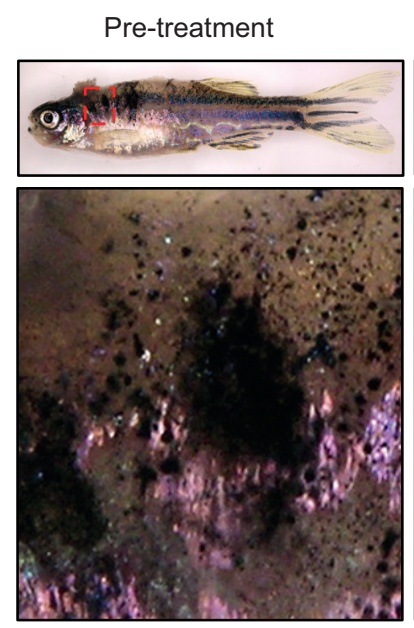

Vemurafenib Week 2
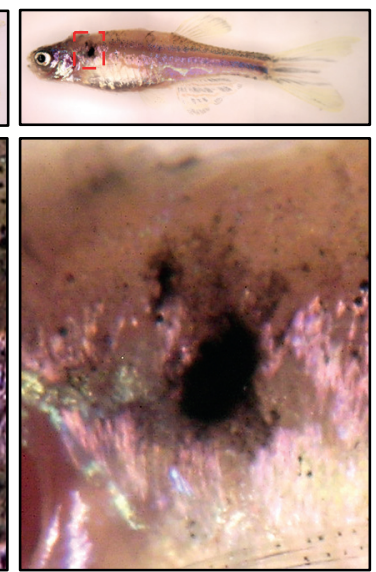

B

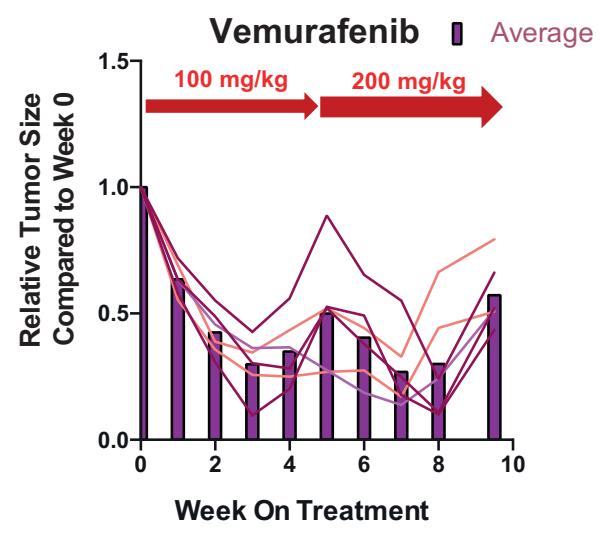

D

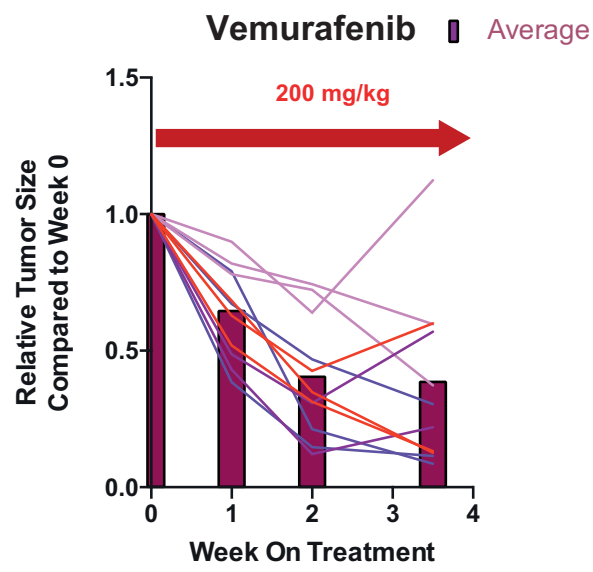


Figure 3. Long-term vemurafenib drug pellet treatment causes acquired drug resistance in zebrafish melanoma.

A. Representative images of $\mathrm{BRAF}^{\mathrm{V} 600 \mathrm{E}}$ zebrafish melanoma before treatment, regressed melanoma, and progressive disease for the animals shown in $\mathbf{B}$.

B. Quantification of melanoma size change each week on $100 \mathrm{mg} / \mathrm{kg}$ vemurafenib pellets or 200 $\mathrm{mg} / \mathrm{kg}$ after dose escalation. Fish receiving vemurafenib pellets $\mathrm{N}=4$. Lesion counts $\mathrm{n}=6$. Each coloured line represents one lesion with the size change tracked over the entire treatment course. Lesions from the same fish share the same colour. The average of all lesions across samples are indicated by column bars.

C. Representative images of $\mathrm{BRAF}^{\mathrm{V} 600 \mathrm{E}}$ zebrafish melanoma before treatment, during melanoma regression, and evidence of recurrent disease while on consistent treatment of $200 \mathrm{mg} / \mathrm{kg}$ vemurafenib.

D. Quantification of melanoma size change each week on $200 \mathrm{mg} / \mathrm{kg}$ vemurafenib pellets. Fish receiving vemurafenib pellets $\mathrm{N}=4$. Lesion counts $n=12$. Each coloured line represents one lesion with the size change tracked over the entire treatment course. Lesions from the same fish share the same colour. The average of all lesions across samples are indicated by column bars. 
A
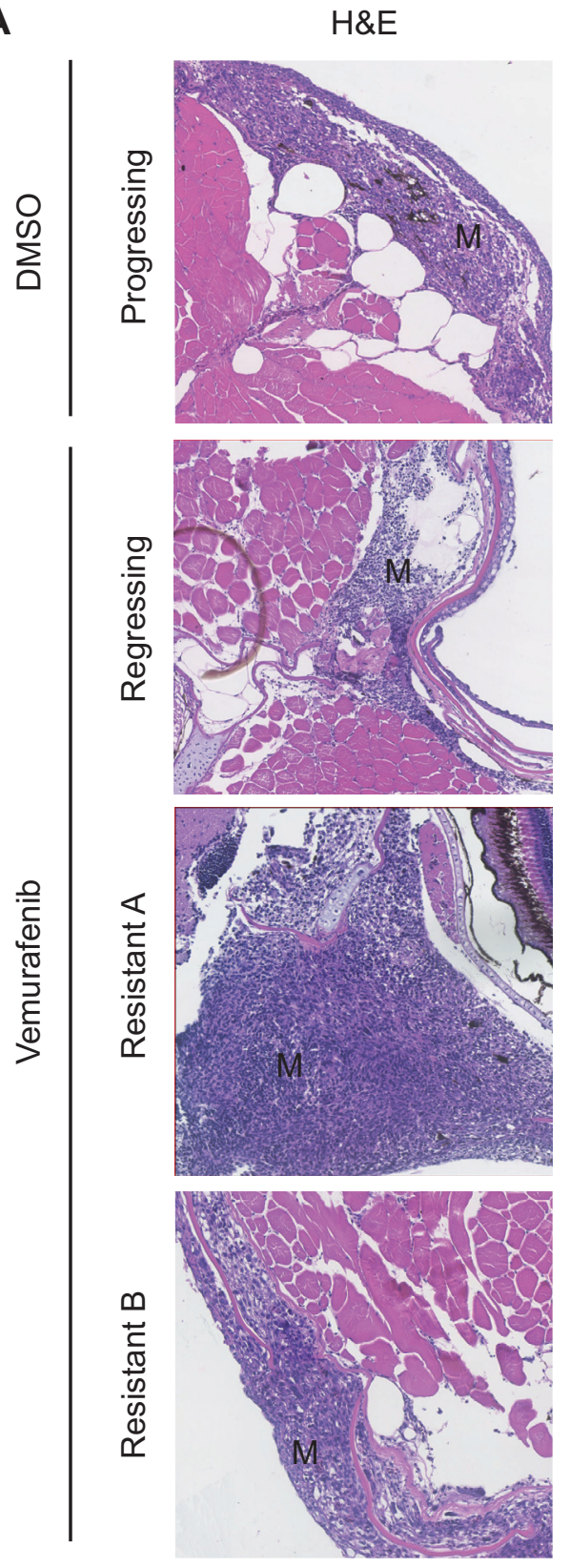

DAPI
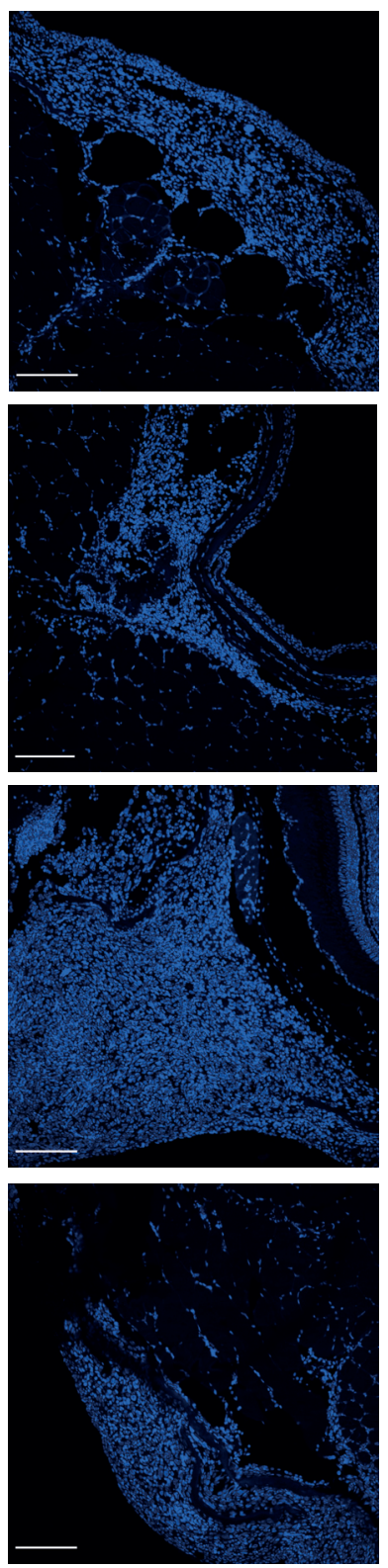

pErk1/2
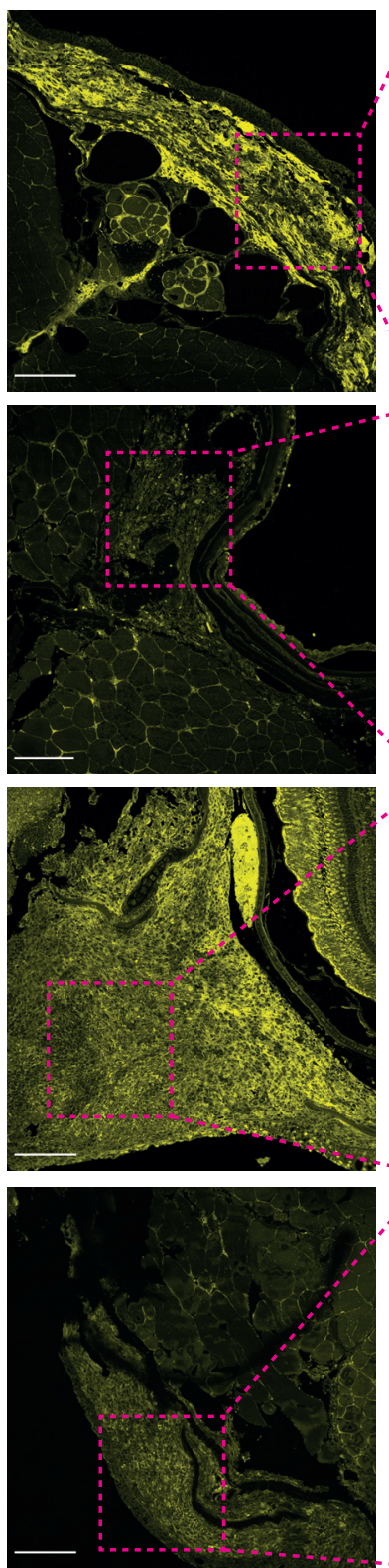

Zoom-in
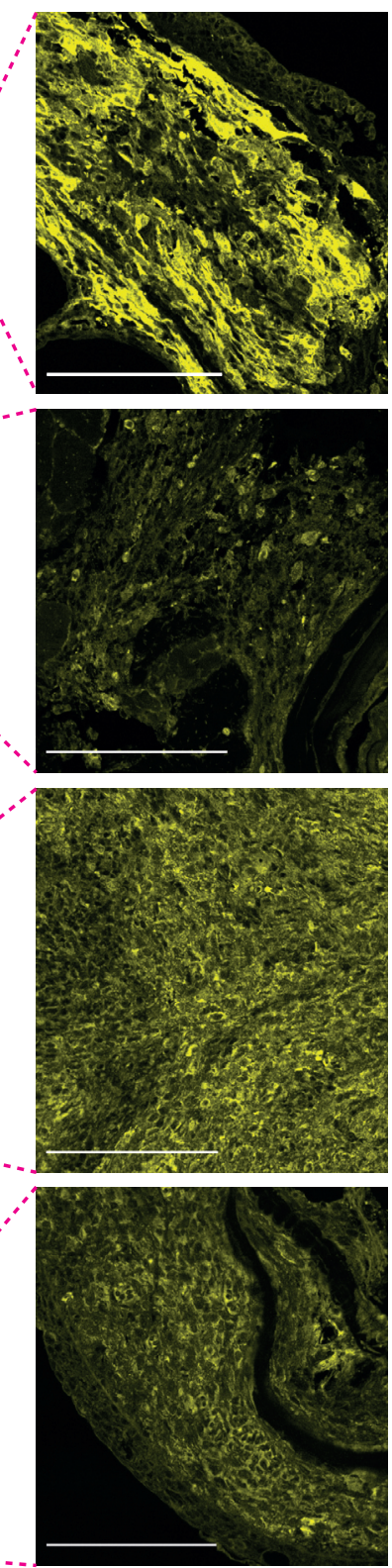

B

C

Phospho-Erk1/2 Levels

Total Erk1/2 Levels

**

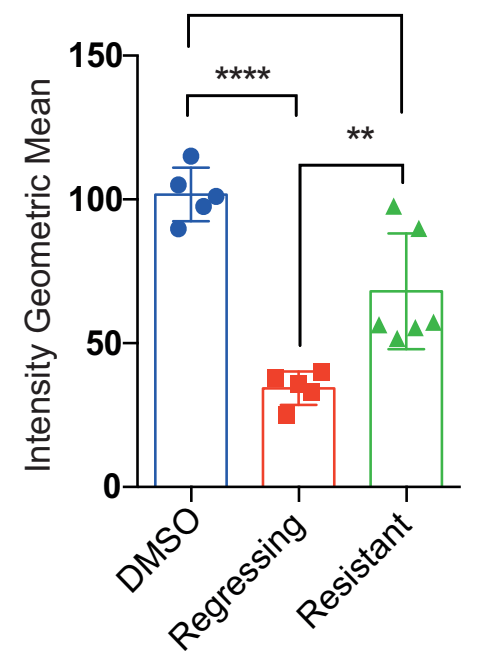

ns

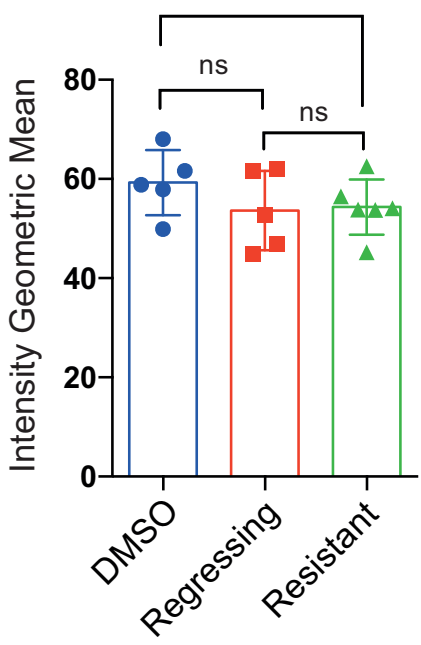


Figure 4. On-target efficacy of vemurafenib drug pellet treatment

A. Representative images of $\mathrm{H} \& \mathrm{E}$ and immunofluorescence staining of BRAF ${ }^{\mathrm{V} 600 \mathrm{E}}$ zebrafish melanoma samples treated with DMSO or Vemurafenib drug pellets. Phospho-Erk1/2 staining in melanoma cells $(\mathrm{M})$ is clearly visible in zoomed regions. Regressing melanomas have reduced phospho-Erk1/2 staining, and the response is varied in Vemurafenib resistant disease. Scale bar $=100 \mu \mathrm{m}$. DMSO treated melanoma sample (week 3, DMSO treatment); melanoma regression sample (week 3, $200 \mathrm{mg} / \mathrm{kg}$ Vemurafenib treatment); Melanoma resistant tumour A and B (week 10; 5-week $100 \mathrm{mg} / \mathrm{kg}$, followed by 5 -weeks $200 \mathrm{mg} / \mathrm{kg}$ Vemurafenib treatment).

B, C. Quantification of immunofluorescence staining intensity of phospho-Erk $1 / 2$ and total Erk1/2 from BRAF ${ }^{\mathrm{V} 600 \mathrm{E}}$ zebrafish melanoma samples treated with DMSO, regressing on Vemurafenib drug pellets, and resistant to Vemurafenib. The DMSO treated samples were collected post 2- or 3-week treatment ( $\mathrm{N}=4$ fish, $\mathrm{n}=5$ lesions). The regressing samples were collected week 3, $200 \mathrm{mg} / \mathrm{kg}$ treatment ( $\mathrm{N}=4$ fish, $\mathrm{n}=5$ lesions). The resistant samples were collected week 10, 5-weeks $200 \mathrm{mg} / \mathrm{kg}$ Vemurafenib treatment escalation course following the initial 5-week $100 \mathrm{mg} / \mathrm{kg}$ Vemurafenib treatment ( $\mathrm{N}=3$ fish, $\mathrm{n}=6$ lesions). (Multiple t-test with Sidak-Bonferroni correction. ns, not significant. ${ }^{* *} \mathrm{p}$ value $<0.01$; ${ }^{* * *} \mathrm{p}$ $<0.0001$ ) 


\section{REFERENCES}

Al-Olabi, L., Polubothu, S., Dowsett, K., Andrews, K.A., Stadnik, P., Joseph, A.P., Knox, R., Pittman, A., Clark, G., Baird, W., et al. (2018). Mosaic RAS/MAPK variants cause sporadic vascular malformations which respond to targeted therapy. J Clin Invest 128, 5185.

Baggiolini, A., Callahan, S.J., Montal, E., Weiss, J.M., Trieu, T., Tagore, M.M., Tischfield, S.E., Walsh, R.M., Suresh, S., Fan, Y., et al. (2021). Developmental chromatin programs determine oncogenic competence in melanoma. Science 373, eabc1048.

Baraban, S.C. (2021). A zebrafish-centric approach to antiepileptic drug development. Dis Model Mech 14.

Ceol, C.J., Houvras, Y., Jane-Valbuena, J., Bilodeau, S., Orlando, D.A., Battisti, V., Fritsch, L., Lin, W.M., Hollmann, T.J., Ferre, F., et al. (2011). The histone methyltransferase SETDB1 is recurrently amplified in melanoma and accelerates its onset. Nature 471, 513-517.

Chang, C.T., Doerr, K.M., and Whipps, C.M. (2017). Antibiotic treatment of zebrafish mycobacteriosis: tolerance and efficacy of treatments with tigecycline and clarithromycin. J Fish Dis $40,1473-1485$.

Chapman, P.B., Hauschild, A., Robert, C., Haanen, J.B., Ascierto, P., Larkin, J., Dummer, R., Garbe, C., Testori, A., Maio, M., et al. (2011). Improved survival with vemurafenib in melanoma with BRAF V600E mutation. N Engl J Med 364, 2507-2516.

Dang, M., Henderson, R.E., Garraway, L.A., and Zon, L.I. (2016). Long-term drug administration in the adult zebrafish using oral gavage for cancer preclinical studies. Dis Model Mech 9, 811820.

Howe, K., Clark, M.D., Torroja, C.F., Torrance, J., Berthelot, C., Muffato, M., Collins, J.E., Humphray, S., McLaren, K., Matthews, L., et al. (2013). The zebrafish reference genome sequence and its relationship to the human genome. Nature 496, 498-503. 
Kaufman, C.K., Mosimann, C., Fan, Z.P., Yang, S., Thomas, A.J., Ablain, J., Tan, J.L., Fogley, R.D., van Rooijen, E., Hagedorn, E.J., et al. (2016). A zebrafish melanoma model reveals emergence of neural crest identity during melanoma initiation. Science 351, aad2197.

Kinkel, M.D., Eames, S.C., Philipson, L.H., and Prince, V.E. (2010). Intraperitoneal injection into adult zebrafish. J Vis Exp.

Kitcher, S.R., Kirkwood, N.K., Camci, E.D., Wu, P., Gibson, R.M., Redila, V.A., Simon, J.A., Rubel, E.W., Raible, D.W., Richardson, G.P., et al. (2019). ORC-13661 protects sensory hair cells from aminoglycoside and cisplatin ototoxicity. JCI Insight 4.

Larkin, J., Ascierto, P.A., Dreno, B., Atkinson, V., Liszkay, G., Maio, M., Mandala, M., Demidov, L., Stroyakovskiy, D., Thomas, L., et al. (2014). Combined vemurafenib and cobimetinib in BRAFmutated melanoma. N Engl J Med 371, 1867-1876.

Li, D., March, M.E., Gutierrez-Uzquiza, A., Kao, C., Seiler, C., Pinto, E., Matsuoka, L.S., Battig, M.R., Bhoj, E.J., Wenger, T.L., et al. (2019). ARAF recurrent mutation causes central conducting lymphatic anomaly treatable with a MEK inhibitor. Nat Med 25, 1116-1122.

Lister, J.A., Capper, A., Zeng, Z., Mathers, M.E., Richardson, J., Paranthaman, K., Jackson, I.J., and Elizabeth Patton, E. (2014). A conditional zebrafish MITF mutation reveals MITF levels are critical for melanoma promotion vs. regression in vivo. J Invest Dermatol 134, 133-140.

Long, G.V., Stroyakovskiy, D., Gogas, H., Levchenko, E., de Braud, F., Larkin, J., Garbe, C., Jouary, T., Hauschild, A., Grob, J.J., et al. (2015). Dabrafenib and trametinib versus dabrafenib and placebo for Val600 BRAF-mutant melanoma: a multicentre, double-blind, phase 3 randomised controlled trial. Lancet 386, 444-451.

Luke, J.J., Flaherty, K.T., Ribas, A., and Long, G.V. (2017). Targeted agents and immunotherapies: optimizing outcomes in melanoma. Nat Rev Clin Oncol 14, 463-482. 
MacRae, C.A., and Peterson, R.T. (2015). Zebrafish as tools for drug discovery. Nat Rev Drug Discov 14, 721-731.

Mandelbaum, J., Shestopalov, I.A., Henderson, R.E., Chau, N.G., Knoechel, B., Wick, M.J., and Zon, L.I. (2018). Zebrafish blastomere screen identifies retinoic acid suppression of MYB in adenoid cystic carcinoma. J Exp Med 215, 2673-2685.

Manzano, J.L., Layos, L., Buges, C., de Los Llanos Gil, M., Vila, L., Martinez-Balibrea, E., and Martinez-Cardus, A. (2016). Resistant mechanisms to BRAF inhibitors in melanoma. Ann Transl Med 4, 237.

Marine, J.C., Dawson, S.J., and Dawson, M.A. (2020). Non-genetic mechanisms of therapeutic resistance in cancer. Nat Rev Cancer 20, 743-756.

North, T.E., Goessling, W., Walkley, C.R., Lengerke, C., Kopani, K.R., Lord, A.M., Weber, G.J., Bowman, T.V., Jang, I.H., Grosser, T., et al. (2007). Prostaglandin E2 regulates vertebrate haematopoietic stem cell homeostasis. Nature 447, 1007-1011.

Patton, E.E., Mueller, K.L., Adams, D.J., Anandasabapathy, N., Aplin, A.E., Bertolotto, C., Bosenberg, M., Ceol, C.J., Burd, C.E., Chi, P., et al. (2021a). Melanoma models for the next generation of therapies. Cancer Cell 39, 610-631.

Patton, E.E., Widlund, H.R., Kutok, J.L., Kopani, K.R., Amatruda, J.F., Murphey, R.D., Berghmans, S., Mayhall, E.A., Traver, D., Fletcher, C.D., et al. (2005). BRAF mutations are sufficient to promote nevi formation and cooperate with p53 in the genesis of melanoma. Curr Biol 15, 249-254.

Patton, E.E., Zon, L.I., and Langenau, D.M. (2021b). Zebrafish disease models in drug discovery: from preclinical modelling to clinical trials. Nat Rev Drug Discov 20, 611-628. 
Proietti, I., Skroza, N., Bernardini, N., Tolino, E., Balduzzi, V., Marchesiello, A., Michelini, S., Volpe, S., Mambrin, A., Mangino, G., et al. (2020). Mechanisms of Acquired BRAF Inhibitor Resistance in Melanoma: A Systematic Review. Cancers (Basel) 12.

Pugach, E.K., Li, P., White, R., and Zon, L. (2009). Retro-orbital injection in adult zebrafish. J Vis Exp.

Rambow, F., Rogiers, A., Marin-Bejar, O., Aibar, S., Femel, J., Dewaele, M., Karras, P., Brown, D., Chang, Y.H., Debiec-Rychter, M., et al. (2018). Toward Minimal Residual Disease-Directed Therapy in Melanoma. Cell 174, 843-855 e819.

Ribas, A., Gonzalez, R., Pavlick, A., Hamid, O., Gajewski, T.F., Daud, A., Flaherty, L., Logan, T., Chmielowski, B., Lewis, K., et al. (2014). Combination of vemurafenib and cobimetinib in patients with advanced BRAF(V600)-mutated melanoma: a phase $1 \mathrm{~b}$ study. Lancet Oncol 15, 954-965.

Robert, C., Karaszewska, B., Schachter, J., Rutkowski, P., Mackiewicz, A., Stroiakovski, D., Lichinitser, M., Dummer, R., Grange, F., Mortier, L., et al. (2015). Improved overall survival in melanoma with combined dabrafenib and trametinib. N Engl J Med 372, 30-39.

Shen, S., Faouzi, S., Souquere, S., Roy, S., Routier, E., Libenciuc, C., Andre, F., Pierron, G., Scoazec, J.Y., and Robert, C. (2020a). Melanoma Persister Cells Are Tolerant to BRAF/MEK Inhibitors via ACOX1-Mediated Fatty Acid Oxidation. Cell Rep 33, 108421.

Shen, S., Vagner, S., and Robert, C. (2020b). Persistent Cancer Cells: The Deadly Survivors. Cell 183, 860-874.

Sosman, J.A., Kim, K.B., Schuchter, L., Gonzalez, R., Pavlick, A.C., Weber, J.S., McArthur, G.A., Hutson, T.E., Moschos, S.J., Flaherty, K.T., et al. (2012). Survival in BRAF V600-mutant advanced melanoma treated with vemurafenib. N Engl J Med 366, 707-714.

Travnickova, J., and Patton, E.E. (2021). Deciphering Melanoma Cell States and Plasticity with Zebrafish Models. J Invest Dermatol 141, 1389-1394. 
Travnickova, J., Wojciechowska, S., Khamseh, A., Gautier, P., Brown, D.V., Lefevre, T., Brombin, A., Ewing, A., Capper, A., Spitzer, M., et al. (2019). Zebrafish MITF-Low Melanoma Subtype Models Reveal Transcriptional Subclusters and MITF-Independent Residual Disease. Cancer Res 79, 5769-5784.

Venkatesan, A.M., Vyas, R., Gramann, A.K., Dresser, K., Gujja, S., Bhatnagar, S., Chhangawala, S., Gomes, C.B.F., Xi, H.S., Lian, C.G., et al. (2018). Ligand-activated BMP signaling inhibits cell differentiation and death to promote melanoma. J Clin Invest 128, 294-308.

Wang, L., Leite de Oliveira, R., Huijberts, S., Bosdriesz, E., Pencheva, N., Brunen, D., Bosma, A., Song, J.Y., Zevenhoven, J., Los-de Vries, G.T., et al. (2018). An Acquired Vulnerability of Drug-Resistant Melanoma with Therapeutic Potential. Cell 173, 1413-1425 e1414.

White, R.M., Cech, J., Ratanasirintrawoot, S., Lin, C.Y., Rahl, P.B., Burke, C.J., Langdon, E., Tomlinson, M.L., Mosher, J., Kaufman, C., et al. (2011). DHODH modulates transcriptional elongation in the neural crest and melanoma. Nature 471, 518-522.

Yan, C., Brunson, D.C., Tang, Q., Do, D., Iftimia, N.A., Moore, J.C., Hayes, M.N., Welker, A.M., Garcia, E.G., Dubash, T.D., et al. (2019). Visualizing Engrafted Human Cancer and Therapy Responses in Immunodeficient Zebrafish. Cell 177, 1903-1914 e1914.

Yu, P.B., Hong, C.C., Sachidanandan, C., Babitt, J.L., Deng, D.Y., Hoyng, S.A., Lin, H.Y., Bloch, K.D., and Peterson, R.T. (2008). Dorsomorphin inhibits BMP signals required for embryogenesis and iron metabolism. Nat Chem Biol 4, 33-41. 